\title{
\begin{tabular}{l|l} 
POLITIQUES \& & Politiques et management public
\end{tabular}

\section{Les démarches locales de performance publique face à la LOLF : mimétisme ou innovation?}

The local public performance process and the LOLF: mimicry or innovation?

\section{Christophe Maurel, David Carassus et Damien Gardey}

\section{(2) OpenEdition}

\section{Journals}

Édition électronique

URL : http://journals.openedition.org/pmp/4423

ISSN : 2119-4831

\section{Éditeur}

Institut de Management Public (IDPM)

Édition imprimée

Date de publication : 15 décembre 2011

Pagination : 417-442

ISSN : 0758-1726

Référence électronique

Christophe Maurel, David Carassus et Damien Gardey, « Les démarches locales de performance publique face à la LOLF : mimétisme ou innovation? », Politiques et management public [En ligne], Vol 28/4 | 2011, mis en ligne le 11 avril 2014, consulté le 30 avril 2019. URL : http:// journals.openedition.org/pmp/4423 


\title{
Les démarches locales de performance publique face à la LOLF : mimétisme ou innovation?
}

\author{
Christophe Maurel ${ }^{a *}$, David Carassus $^{b}$ et Damien Gardey ${ }^{c}$ \\ ${ }^{a}$ Maître de conférences à Université du Maine, GAINS-ARGUMANS (EA 2167) \\ ${ }^{b}$ Maître de conférences / HDR, Université de Pau et des Pays de l'Adour, \\ IAE/Centre de Recherche et d'Études en Gestion \\ c Professeur de finance - Groupe ESC Pau
}

\section{Résumé}

Les démarches de modernisation dans le secteur public ces démarches peuvent s'appuyer sur des modèles existants d'évaluation et de mesure de la performance publique, ou être expérimentées sur des bases innovantes. Ainsi, à travers cinq Conseils Généraux, nous étudions le contenu des démarches de performance afin d'apprécier le degré d'influence du cadre de la loi organique relative aux lois de finances (LOLF). Les observations montrent un contenu instrumental proche de la LOLF, même si les objectifs poursuivis semblent plus globaux, et queles collectivitésétudiées mettenten œuvredesmodalités spécifiques.Ces résultats soutiennent un modèle global de la performance publique, adaptable aux collectivités locales, et militent pour la mise en œuvre de facteurs clés spécifiques favorisant la réussite de ces démarches.

(c) 2011 IDMP/Lavoisier SAS. Tous droits réservés

Mots clés : collectivité locale, contrôle de gestion, évaluation, performance publique

\section{Abstract}

The local public performance process and the LOLF: mimicry or innovation? The initiatives of modernization in the public sector can build on existing models of evaluation and measurement public performance, or be tested on innovative bases. Thus, through five local authorities, we study the content of performance-based approaches to assess the degree of influence of the Constitutional Bylaw on Budget Acts. The findings show the content is close to the LOLF tools, even if the goals seem more global, and that the implementation in the local authorities is specific. These results argue for an overall model of public performance

(c) 2011 IDMP/Lavoisier SAS. Tous droits réservés

Keywords: local authority, management, performance, public

*Auteur correspondant : christophe.maurel@univ-lemans.fr doi:10.3166/pmp.28.417-442 @ 2011 IDMP/Lavoisier SAS. Tous droits réservés 


\section{Introduction}

L'essor de la gestion de la performance dans le secteur public est le résultat d'un processus de réformes entreprises dans les pays développés durant les années 1990. Elle implique que la culture de moyens qui prévalait jusque-là soit abandonnée au profit de « la culture de résultats » (Bied-Charreton, 2006). Selon cet auteur, les deux tiers des pays membres de l'Organisation de coopération et de développement économiques (OCDE) l'ont déjà adoptée. "Ils sont désormais conduits à évaluer l'action publique et à mettre en place des programmes annuels de performance ». Même s'il ne semble pas exister de modèle unique, des tendances convergentes se dessinent dans les processus de modernisation, motivées par l'assainissement des finances publiques, la rénovation de la procédure budgétaire, et des cadres plus souples pour la politique de ressources humaines. Ces démarches de modernisation ne concernent, le plus souvent, que l'administration d'Etat, mais elles intéressent aussi les collectivités territoriales. Cependant, ces dernières ne semblent pas disposer d'un cadre formalisé de réforme pour initier une gestion de la performance, à l'image de la France au niveau étatique avec le vote de la Loi Organique Relative aux Lois de Finances (LOLF), en 2001, concernant les structures relevant de l'Etat.

Sans méconnaître la nécessité d'une gestion de la performance et le risque de plaquer un modèle sur une réalité administrative insuffisamment transformée, des collectivités se lancent, depuis quelques années, dans des démarches de performance adaptées à leur contexte, que cela soit aux Etats-Unis, en Australie, en Italie (Guthrie et Farneti, 2008) ou, maintenant, en France. Cependant, si la notion de performance est au cœur des politiques de réformes managériales publiques, son analyse est délicate du fait des deux niveaux de réalité auxquels elle renvoie : la performance peut être l'objectif (mettre en œuvre un système de gestion de la performance), mais aussi le moyen des réformes publiques (créer des objectifs de performance).

Ainsi, nous analysons ici les pratiques des premières démarches de performance initiées par les collectivités françaises de manière volontaire. Naturellement, l'absence de cadre formel rend potentielles les expériences diverses, mais cette situation est reconnue et souhaitée par les initiateurs de la LOLF dans un rapport remis au Gouvernement en novembre 2006 : «La LOLF inspire de nombreuses collectivités locales souhaitant moderniser leur gestion. Il ressort des expérimentations en cours que ces collectivités adoptent, à partir de principes communs de gestion orientée vers les résultats, des organisations très différentes les unes des autres en raison de leurs spécificités. Il est préférable d'accompagner ces expérimentations plutôt que de tenter de les formater dans un moule commun qui découragerait leurs promoteurs » (Lambert et Migaud, 2006). Si le rapport précédent conclut qu'une LOLF locale serait inopportune, il propose par contre de favoriser l'extension de ses principes aux collectivités territoriales. Selon le Conseil de l'Europe (2005), quatre pays - le Royaume Uni, le Danemark, la Turquie et la Roumanie - ont ainsi déjà confié au gouvernement central la majeure partie des responsabilités en matière de contrôle et de gestion de la performance des collectivités locales.

L'intérêt de cette recherche réside alors dans l'étude du contenu des démarches locales de performance et leurs différences avec le cadre de référence proposé par la LOLF ; en d'autres termes, les démarches de performance récemment initiées par les collectivités locales françaises correspondent-elles à des innovations ou peuvent-elles être analysées comme le résultat d'un mimétisme vis-à-vis des principes étatiques LOLFiques ? 
Afin de pouvoir analyser les démarches de performance locale, l'étude s'appuie sur les données observées dans différents conseils généraux (CG) au cours de l'année 2009. Nous centrons l'analyse sur le contenu des démarches de performance et non sur le processus de mise en œuvre, pour lequel de nombreux travaux portant sur l'analyse d'une réforme ou d'un changement organisationnel existent. Une recherche sur le contenu sert à mettre en évidence de quoi se compose l'objet étudié. Elle n'a pas pour objectif l'explication de l'objet étudié au sens d'une recherche de causalité, mais plutôt sa description pour améliorer la compréhension (Thiétart et al., 1999, p.110). Par ailleurs, pour comparer le contenu des démarches de performance entre elles, nous retenons une seule strate de collectivité, en l'espèce les conseils généraux (CG). Au sein de chaque CG, les données issues des documents et des entretiens sont synthétisées selon le processus de réduction décrit par Miles et Huberman (2003) en faisant émerger des thèmes transversaux d'analyse. Les résultats montrent un certain mimétisme avec la LOLF, notamment au niveau des outils utilisés, alors que les modalités de mise en œuvre des démarches locales d'amélioration de la performance apparaissent spécifiques.

Avant de présenter nos observations et de répondre à notre problématique, le cadre des démarches de performance publique est exposé dans une première partie, puis les résultats observés dans des conseils généraux sont proposés.

\section{L'environnement des démarches de performance publique locale}

Si la gestion de la performance dans le secteur public s'insère principalement dans le cadre théorique du New Public Management, le cadre des pratiques apparaît plus divers avec l'adaptation de modèles génériques. Par ailleurs, au niveau local, les collectivités bénéficient de premiers retours d'expériences quant aux instruments mis en œuvre, qui permettent de s'interroger sur le mimétisme ou l'innovation managériale.

\subsection{Les principaux modèles de performance et la LOLF}

Dans le secteur public, les idées portant sur la gestion de la performance s'inscrivent dans le courant théorique de la nouvelle gestion publique (NGP) ou New Public Management (NPM), comportant lui-même de nombreuses approches (Hood, 1995). En effet, l'évaluation de la performance, comme des résultats, sont au cœur des approches développées dans le sillage du NPM (Osborne et Gaebler, 1993). La NGP recommande de promouvoir la qualité des services fournis aux citoyens-usagers, sur la base d'une évaluation de leurs besoins, puis d'une évaluation des prestations effectivement fournies par l'organisation publique, au moyen d'indicateurs appropriés. Dans ce sens, Möncks (1998) identifie trois modèles-types de NGP : le modèle de l'efficience, le modèle de la décentralisation, et le modèle participatif (lui-même associé à un modèle de l'excellence par la qualité délivrée). Ces modèles types sont plus ou moins adaptés par les Etats, dans les années 1990, pour moderniser les structures publiques.

Parallèlement, Boyne (2002) indique que les organisations publiques peuvent faire référence à trois principaux modèles de performance. Le premier type de modèle est utilisé dans le secteur privé et représente la performance par le triptyque Objectifs-Moyens-Résultats. Le second type de modèle intègre la double fonction de production des organisations 
publiques développée par Gibert (1988), qui distingue la fonction interne caractérisant toute organisation et la fonction de production externe visant à modifier l'environnement. En différenciant les produits ou résultats de l'action (outputs) et les impacts des politiques publiques (outcomes), de nombreux modèles s'insèrent alors dans cette approche (Bouckaert, 2006 ; Hatry, 1999 ; Osborne et Plastrik, 2000). Le troisième type de modèle est fondé sur les «approches partenariales » de la performance, notamment à travers les adaptations du Balanced Scorecard (BSC) de Kaplan et Norton (1996) et du Cadre d'Auto-Evaluation des Fonctions Publiques (CAF) ${ }^{1}$ (2006). Ces modèles, qui détaillent les résultats de l'action publique selon plusieurs dimensions, sont ceux qui se développent dans les collectivités suivant Guthrie et Farneti (2008) ou Chauvey (2005).

Cependant, que ce soit les modèles de représentation ou de mesure de la performance, les démarches mises en œuvre au niveau des Etats ou des collectivités sont généralement inspirées de plusieurs soubassements théoriques. C'est ainsi le cas de la démarche de performance publique initiée en France avec le vote en août 2001 de la loi organique relative aux lois de finances (LOLF) concernant les administrations d'Etat. En effet, la LOLF s'insère dans le mouvement général d'impulsion d'une culture du résultat au sein des administrations. Selon le Guide méthodologique pour l'application de la LOLF (Coll., 2004), la démarche LOLF s'appuie sur la présentation d'une stratégie associée à des programmes. Puis, des objectifs en découlent et sont évalués par des indicateurs, faisant l'objet d'un reporting annuel aux décideurs. Aussi, le budget est organisé autour de la finalité des dépenses en trois niveaux : Mission, Programme, Action (MPA). Il est voté en fonction d'objectifs précis sur lesquels s'engage l'exécutif. Un choix d'objectifs « équilibrés » doit correspondre, selon le même guide méthodologique (Coll., 2004), aux attentes des citoyens (objectifs relatifs à l'efficacité socio-économique), des usagers (la qualité de service) et des contribuables (l'efficience). Ces objectifs dits stratégiques ont vocation à être déclinés en objectifs opérationnels, instruments privilégiés de pilotage des services. Ce travail de déclinaison implique le responsable de programme, les responsables des services et les agents et permet de déterminer des indicateurs et d'élaborer des tableaux de bord.

Outre la dimension instrumentale, la LOLF présente aussi des pratiques de gestion par la performance. Le guide méthodologique propose, en effet, de délimiter des centres de prestations à l'intérieur de l'administration et en confier la direction à des gestionnaires publics bénéficiant d'une autonomie accrue par rapport au système traditionnel pour atteindre les objectifs fixés. Le pilotage de la gestion se fait en fonction des résultats attendus. Il est assuré par les responsables de programmes, par les responsables de services et les agents.

Nous pouvons remarquer que parmi ces dispositions de la LOLF, des éléments sont déjà applicables aux collectivités locales. Dans le cadre de la procédure budgétaire, les informations généralement fournies pour mener à bien au niveau local le débat d'orientation budgétaire sont le reflet de celles imposées au niveau national par l'article 48 de la LOLF. Ainsi, la présentation fonctionnelle du budget des collectivités est proche de la nouvelle présentation budgétaire de l'État issue de la LOLF. De plus, les modalités offertes au niveau du vote des budgets locaux comportent déjà une certaine souplesse dans l'utilisation des crédits, ainsi qu'une pluriannualité. Par ailleurs, la logique de transparence, rappelée dans

\footnotetext{
' Développé par l'European Foundation for Management Quality (EFQM) et I'Institut Européen d'Administration Publique (IEAP). Guide d'utilisation du CAF, version 2006.
} 
la LOLF, est déjà intégrée dans les dispositions qui s'imposent aux collectivités locales à travers le décret du 29 décembre $1962^{2}$.

Aussi, certaines collectivités locales se sont déjà appuyées sur le contexte favorable à une démarche de performance impulsée par la LOLF pour initier ou approfondir leur démarche (Grail et al., 2006).

\subsection{Les expériences des premières démarches de performance}

Au plan international, parmi les premières tentatives de démarches locales de performance publique se trouvent les grandes villes anglo-saxonnes telles que Charlotte, Melbourne, Sydney, Auckland, ainsi que le niveau fédéral aux États-Unis ou en Australie (English et Guthrie, 1997). Ces premières initiatives visent à améliorer l'efficience de l'organisation. De plus, progressivement, l'approche de la performance proposée par Kaplan et Norton (1996) avec le Balanced Scorecard (BSC) semble se généraliser dans les administrations de l'Amérique du nord (Charlotte), du Québec, dans les villes australiennes de Brisbane et Melbourne (Yetano, 2009), mais aussi en Europe (Manchester, Vienne, Sienne - Farneti, 2006 ; Guthrie et Farneti, 2008). Cette situation peut s'expliquer par la souplesse d'utilisation du BSC dont les deux auteurs ont revendiqué l'adaptation à la majorité des organisations.

Cependant, des études montrent que la transposition de l'outil est difficile dans le secteur public (Benzerafa, 2007), en raison, par exemple, de la prise en compte des attentes et des contributions des acteurs constitutifs de l'action publique de la collectivité (Gibert, 2002). D'autres modèles de performance utilisés par les collectivités font, eux, référence au Cadre d'Autoévaluation des Fonctions publiques ${ }^{3}$, ou au modèle de Bouckaert et Pollitt (2004) lesquels articulent différents dispositifs de pilotage de la performance pour schématiser la performance publique. Ainsi, les études de cas présentées dans les colloques de l'Association Finances-Gestion-Evaluation des collectivités (AFIGESE-CT) et de Ville-Management ${ }^{4}$ montrent que différentes collectivités, en fonction de leur histoire, leurs priorités et leurs moyens, définissent des objectifs de performance.

Aussi, l'approche de la performance est relativement hétérogène, mais, dans tous les cas, la préoccupation des collectivités est unanime. Cette situation peut en effet s'expliquer par différents enjeux (Carassus et al., 2010), à savoir un enjeu financier, par la mise en place d'une démarche de performance dans un contexte de contrainte budgétaire, et/ou un enjeu organisationnel, pour clarifier les missions et responsabilité de chacun, et/ou un enjeu démocratique, pour améliorer la lisibilité du budget de la collectivité et des actions envers les administrés. Ces différents enjeux vont le sens d'un modèle complet d'évaluation de la performance des gouvernements locaux (Kloot et Martin, 2000). Or, même si Edwards et Thomas (2005) avancent que l'outil proposé par Kaplan et Norton permet, par adaptations, de traduire la performance de la ville d'Atlanta, il nous semble que le BSC seul, comme

\footnotetext{
${ }^{2}$ De même, le principe de sincérité inscrit dans les instructions comptables des collectivités est aussi rappelé dans l'ordonnance du 26 août 2005 relative à la simplification et à l'amélioration des règles budgétaires et comptables.

${ }^{3}$ Farneti, supra, évoque des adaptations du CAF en Italie ; le guide d'utilisation du CAF mentionne ainsi 1000 expériences en Europe dans les structures publiques (administrations, collectivités, entreprises publiques...).

${ }^{4} \mathrm{Cf}$. http://www.ville-management.org/index2.htm (rubrique Workshop).
} 
la « cascade de la performance » proposée dans la LOLF, ne répond pas aux besoins des collectivités françaises désirant un outil englobant qui permet aux acteurs d'avoir une vision générale de l'organisation. Celle-ci apparaît en effet à travers les problématiques de sécurité, de développement territorial, d'environnement, mises en parallèle avec les problématiques financières, organisationnelles, économiques et sociales auxquelles sont confrontées les collectivités. Ces dernières peuvent alors recourir à des modèles de performance existants ou bien initier des démarches plus ou moins innovantes.

\subsection{Les facteurs explicatifs des pratiques volontaires des collectivités et les facteurs de succès des démarches expérimentées}

Comme dans tout changement au sein d'organisations publiques, entendu comme « une modification, observable dans le temps, d'une situation existante et qui affecte l'organisation ou le fonctionnement d'une entité, d'une manière non provisoire » (Amiel et al., 1998, p. 217), deux voies s'offrent aux collectivités afin de sélectionner le contenu de leur démarche de performance : celle de l'imitation et celle de l'innovation (Metcalfe, in Bouckaert et Halachmi, 1995).

Concernant la première de ces voies, celle de l'imitation, elle consisterait à transposer les principes et méthodes recommandées par le guide méthodologique de la LOLF, dont les exemples dans les administrations sont déjà nombreux. Le recours à ce mimétisme pourrait alors s'expliquer, selon la théorie néo-institutionnelle (DiMaggio et Powell, 1983), à partir des formes d'isomorphisme. En effet, l'imitation peut être recherchée pour la légitimité, en montrant que la collectivité est aussi performante qu'une administration, ou comme réponse à des pressions externes, par exemple d'experts, de consultants, ou de directions administratives. Par ailleurs, les logiques d'imitation font appel à la notion de mode, c'est-à-dire l'attrait pour les nouveaux outils, nouvelles méthodes et pratiques de gestion. Cependant, la transposition directe peut se heurter à des limites identitaires et culturelles, pour garder une libre administration en n'intégrant pas les règles introduites dans l'administration d'Etat, ou à des défis techniques, par la mise en place de moyens conséquents qui semblent nécessaire pour respecter les principes de la LOLF. En outre, Jick (1993) explique que toute démarche de changement doit respecter un principe de contingence, mais aussi éviter les risques associés au fait d'appliquer dans un contexte des formules de changement qui ont fait leur preuve dans d'autres situations. Aussi, les possibilités de transfert de la LOLF dans les collectivités nous semblent reposer sur les principes d'adaptation, de contingence et de capitalisation sur les expériences menées.

A l'opposé, la seconde voie de changement, celle de l'innovation, consisterait à recourir à une démarche de performance fondée sur un contenu distinct des formes actuelles. La volonté des élus locaux ou des contraintes et opportunités locales peuvent expliquer ce choix. Néanmoins, nous sommes conscients, comme Bouckaert et Halachmi (1995, p.13) l'indiquent, que souvent les voies de l'imitation et de l'innovation s'entremêlent dans les changements introduits dans les collectivités. On aboutit alors à une «innovation mimétique », qui associe l'importation de solutions efficaces observés dans le champ organisationnel et le développement de changements spécifiques adaptés au contexte de l'organisation. En ce sens, les démarches locales de performance pourraient être considérées comme des pratiques volontaires représentant à la fois un changement conceptuel comparé au cadre légal de la LOLF et une adaptation de démarches existantes. 
Les démarches locales de performance visent un ou des apprentissages dans les collectivités. Cependant pour que les apprentissages aient lieu, des facteurs de réussite apparaissent nécessaires. Carassus et Favoreu (2005), dans leur étude de l'application de la LOLF aux collectivités locales, expliquent qu'une mise en oeuvre réussie des principes de la LOLF passe par le principe d'adaptation de la méthode, le principe de progressivité de l'introduction de la démarche, le principe d'expérimentation et de volontariat, mais aussi le soutien et l'implication tant des élus politiques que des responsables administratifs. Ces éléments sont repris dans la littérature à travers différents travaux (Guillaume et al., 2002; Huteau, 2008 ; Lambert et Migaud, 2005 ; Paquin et Tremblay 1997 ). Au global, ils permettent, tout d'abord, de distinguer les facteurs de succès organisationnels d'une démarche locale de performance, à savoir bénéficier d'un soutien et d'une implication politique des élus de la collectivité et de la direction générale, créer des interactions entre les différents acteurs de la collectivité et créer un groupe projet autour de cette démarche (comité de pilotage, etc.). Ensuite, ils dégagent les facteurs de succès méthodologiques, tels que prévoir un plan pluriannuel d'implémentation dans le cadre d'une démarche en plusieurs étapes (principe de progressivité de la démarche), créer et enraciner progressivement le changement culturel induit par le passage à une démarche locale de performance et de résultat, ainsi qu'adapter la démarche locale de performance aux caractéristiques de la collectivité locale concernée.

Au final, cette présentation des contextes des démarches de performances locales (DPL) nous permet d'obtenir une grille de lecture exposant les caractéristiques du contenu des DPL par rapport aux principes et outils LOLFiques. Les caractéristiques des DPL sont ainsi abordées par la suite selon les cinq critères qui suivent ${ }^{5}$, où nous détaillons les recommandations de la LOLF :

- le périmètre d'intervention. Dans la démarche de performance de l'administration, la LOLF vise à passer à « une logique d'objectifs et de résultats pour gérer par la performance » l'ensemble de la structure concernée, c'est-à-dire les activités menées et le personnel.

- les modalités de mise en œuvre, autour des facteurs de succès organisationnels et méthodologiques. Dans le cadre de sa mise en œuvre, la LOLF a été votée par l'ensemble des élus puis portée par l'administration selon un calendrier serré et des indicateurs imposés à renseigner dans le cadre d'un reporting ascendant pour les organismes de tutelle.

- les objectifs poursuivis. Dans la LOLF, si la motivation immédiate semble être le renforcement du contrôle parlementaire, trois objectifs sont affichés : la sincérité et la transparence des comptes (abordés dans différents articles avec la notion de lisibilité, dont l'article 30 portant sur les principes généraux de comptabilité), la stabilité ou maitrise des dépenses publiques (articles 27 et 52).

- les concepts mobilisés pour évaluer la performance et les indicateurs associés. Dans la LOLF, les concepts d'objectifs et de résultats sont fréquemment mobilisés pour introduire l'efficacité et l'efficience, appréciés avec trois axes d'analyse : la qualité du service rendu, l'efficacité socio-économique, et l'efficience de la gestion.

- les pratiques promues. Dans le cadre de sa mise en œuvre, la LOLF mentionne le recours aux pratiques de décentralisation (gérer le plus localement possible), ce qui s'associe à une responsabilisation des cadres et des agents, et une contractualisation d'objectifs.

\footnotetext{
${ }^{5}$ Critères basés sur l'analyse de la littérature et des codages d'entretiens effectués avec le logiciel Nvivo détaillés dans la partie méthodologie, infra.
} 
Une synthèse présentée en tableau 1 propose les caractéristiques du cadre de référence de la LOLF vis-à-vis de notre grille d'analyse.

Tableau 1 : Caractéristiques de la LOLF sur les cinq critères

\begin{tabular}{|l|l|l|l|l|l|}
\hline & Périmètre & Modalités & Objectifs & $\begin{array}{l}\text { Concepts } \\
\text { et indicateurs }\end{array}$ & Pratiques promues \\
\hline $\begin{array}{l}\text { LOLF } \\
\text { (référentiel } \\
\text { de l'Etat) }\end{array}$ & $\begin{array}{l}\text { Ensemble de } \\
\text { l'organisation } \\
\text { (activités } \\
\text { et agents) }\end{array}$ & $\begin{array}{l}\text { Fixées et } \\
\text { imposées }\end{array}$ & $\begin{array}{l}\text { la sincérité } \\
\text { et la transparence } \\
\text { des comptes, } \\
\text { la maîtrise } \\
\text { des dépenses } \\
\text { publiques }\end{array}$ & $\begin{array}{l}\text { Notion de résultats; } \\
\text { Indicateurs } \\
\text { relatif à la qualité } \\
\text { du service rendu, } \\
\text { à l'efficacité } \\
\text { socio-économique, } \\
\text { et l'efficience } \\
\text { de la gestion }\end{array}$ & $\begin{array}{l}\text { Décentralisation; } \\
\text { Responsabilisation; } \\
\text { Contractualisation }\end{array}$ \\
\hline
\end{tabular}

Le cadre de cette recherche étant précisé, nous confrontons maintenant cette grille d'analyse à des pratiques empiriques, permettant de définir les démarches locales et volontaires de performance publique.

\section{La caractérisation des démarches volontaires de performance dans les collectivités locales}

Cette caractérisation est assurée en deux temps. Tout d'abord, nous précisons la méthodologie de notre recherche. Ensuite, nous en présentons les résultats.

\subsection{Méthodologie de la recherche}

Dans le cadre de notre méthodologie exploratoire visant à caractériser les démarches de performance menées dans les collectivités au regard de celle menée au niveau de l'Etat, nous mobilisons la méthode des cas multi-sites. En adoptant un positionnement d'observateur, différentes visites ont ainsi été effectuées dans des collectivités françaises.

Pour notre choix des cas, comme l'indique Hlady-Rispal (2002, p.82), «les études de cas multi-sites reposent sur un échantillonnage théorique, ce qui signifie que les cas sont choisis pour des motifs non pas statistiques mais théoriques ». Nous avons retenu des collectivités ayant déjà engagé une démarche de performance, à partir d'informations obtenues de l'AFIGESE$\mathrm{CT}$ et de cadres territoriaux, puis nous avons travaillé avec celles qui acceptaient de nous recevoir. Cet échantillon de convenance nous a cependant permis d'étudier des collectivités ayant initié des démarches spécifiques et, par conséquent, d'obtenir une certaine variété. En effet, les cinq collectivités présentées sont caractérisées par l'adoption d'une démarche de performance, mais aussi par une certaine diversité, puisque deux conseils généraux sont plus grands et urbanisés que la moyenne nationale, deux autres dans les moyennes et le cinquième présente des tailles d'habitants et d'effectifs inférieures aux moyennes nationales. Par ailleurs, cette étude ne présente qu'une seule strate de collectivité, les conseils généraux, afin de mieux pouvoir comparer le contenu des démarches de performance. 
Afin de répondre à notre problématique, deux types de données sont utilisés : des données factuelles (analyse de documents internes) et déclaratives (recueils d'entretiens). Nous avons recueilli ces données lors de visites durant l'année 2009. Aussi, pour cette étude, nous nous appuyons principalement sur les documents afférents aux démarches de performances ${ }^{6}$ et sur des entretiens menés auprès de responsables. En effet, lors des visites, nous avons interrogé les responsables locaux administratifs (directeur général des services, directeur du contrôle, de l'évaluation, des finances, et des ressources humaines) et des élus locaux concernés par les démarches de performance. Si ces rencontres ont fait l'objet d'entretiens semi-directifs, une partie seulement des données recueillies concerne cette étude. En effet, notre guide d'entretien comportait une partie relative au contexte d'impulsion de la démarche de performance, une partie sur les outils et pratiques de gestion (intéressant cette étude), et une autre sur les effets de la démarche, avec une conclusion sur les projets laissant la place à des adaptations avec l'interlocuteur ${ }^{7}$. Chaque entretien a duré entre 45 minutes et deux heures. Ils ont été enregistrés et retranscrits avec une condition d'anonymat. Dans chaque cas, l'observation des documents a permis de s'assurer que les discours étaient «cohérents» avec les actes.

Pour obtenir la grille d'analyse en cinq critères présentée à la fin de partie 1 , les retranscriptions d'entretiens ont été codées dans le logiciel Nvivo en reprenant les thèmes abordés pendant les entretiens, parallèlement au codage manuel de certains documents internes. Le codage final, après réduction des données, a fait ressortir les catégories d'analyse transversales aux démarches de performance des collectivités étudiées : le périmètre retenu, les modalités de la démarche, les concepts et objectifs mesurés, et enfin les instruments mobilisés et les pratiques promues.

Chacun des thèmes est exposé en s'appuyant principalement sur les documents utilisés dans les démarches de performance. Ces données peuvent donc comporter un caractère déclaratif limitant la portée comparative, mais, parallèlement, elles créent le référentiel des expériences menées et sont donc pertinentes pour l'analyse.

\subsection{Présentation des éléments de caractérisation des démarches performances publiques locales mises en œuvre}

Nous avons synthétisé la grille d'analyse comportant les cinq critères en trois thèmes pour faciliter la discussion des résultats. Aussi, le périmètre et les modalités de mise en oeuvre des démarches de performance sont présentés ensemble, puis les concepts retenus et les objectifs en découlant avec les indicateurs associés font l'objet d'un même point. Enfin, les instruments mobilisés et les pratiques promues sont exposés.

\footnotetext{
${ }^{6}$ Les dossiers internes sur les démarches de performance, comportant les lettres d'information, les présentations Powerpoint, les rapports sur les démarches de performance, et les instruments des démarches tels que les tableaux de bord, les projets pluriannuels de performance, les fiches internes aux services, etc. ${ }^{7}$ Les cinq thèmes du guide (Thème 1 : les contextes et enjeux de la démarche ; Thème $2:$ les modalités d'implémentation ; Thème 3 : les outils de la démarche, avec des questions sur les instruments stratégiques, budgétaire et comptable, de gestion du personnel, et outils d'évaluation de la performance ; Thème 4 : les impacts de la démarche; Thème 5 : les évolutions de la démarche et les projets) ont fait l'objet d'entretiens avec les différents directeurs de services cités et parfois des élus locaux, soit au minimum six personnes.
} 


\subsubsection{Le périmètre des démarches de performance et les modalités de mise en œuvre}

Au sein des cinq conseils généraux (CG), sur la base des rapports annuels de performance, nous avons observé des différences notables allant d'un champ d'intervention couvrant la collectivité en général, c'est-à-dire les activités et le personnel, et ses partenaires privilégiés, à un champ restreint aux seules activités jugées pertinentes (certaines politiques publiques).

Ainsi le premier conseil général a initié une démarche de performance englobant l'organisation et ses partenaires. "La collectivité dans son ensemble », selon l'introduction du rapport annuel de performance, est concernée, c'est-à-dire toutes les ressources humaines, financières et matérielles utilisées et les satellites, les usagers, les partenaires économiques et institutionnels. Dans le second CG, les mêmes termes sont utilisés dans le rapport, mais aucune information ne concerne les partenaires de la collectivité. Le troisième CG a lui développé une démarche de performance qui comprend seulement les activités menées par les directions de la collectivité. Dans le $\mathrm{CG} \mathrm{n}^{\circ} 4$, la démarche de performance ne porte que sur certaines activités, les directions administratives n'étant pas concernées, mais aussi sur les agents. Enfin, dans le CG n ${ }^{\circ}$, seules certaines activités sont concernées. Les rapports sur la démarche de performance montrent qu'au moment des visites la moitié des directions (Transports, Education et jeunesse, et Vie sociale) s'inscrivaient dans la démarche.

Nous obtenons donc une certaine variété dans le périmètre des démarches impulsées par les conseils généraux visités.

Tableau 2 : Le périmètre des démarches dans les cinq collectivités

\begin{tabular}{|l|l|l|l|l|l|}
\hline & CG1 & CG2 & CG3 & CG4 & CG5 \\
\hline $\begin{array}{l}\text { Périmètre } \\
\text { (d'après les } \\
\text { rapports de } \\
\text { performances) }\end{array}$ & $\begin{array}{l}\text { Les activités } \\
\text { du CG et des } \\
\text { satellites, } \\
\text { Le personnel }\end{array}$ & $\begin{array}{l}\text { Les activités du CG } \\
\text { (et des satellites), } \\
\text { Le personnel }\end{array}$ & $\begin{array}{l}\text { Les } \\
\text { activités } \\
\text { du CG }\end{array}$ & $\begin{array}{l}\text { Des activités } \\
\text { du CG, } \\
\text { Le personnel }\end{array}$ & $\begin{array}{l}\text { Des } \\
\text { activités } \\
\text { du CG }\end{array}$ \\
\hline
\end{tabular}

Nous pourrions avancer que la date de mise en œuvre de la démarche explique la jeunesse de certaines approches et leur périmètre restreint, mais il s'avère que l'ensemble des CG étudiés a impulsé sa démarche entre 2005 et 2008. Une autre explication de la diversité du périmètre pourrait tenir à l'origine de la décision d'impulsion des démarches de performance. En effet, de nombreuses enquêtes sur le changement dans les collectivités ${ }^{9}$, ou bien sur la mise en place de réformes, admettent qu'une impulsion associant la sphère politique et administrative augmente le succès du changement planifié, d'autant plus s'il impacte de nombreux services, ce qui est le cas des projets transversaux tels que le changement d'instruction comptable, ou, en l'espèce, le projet d'un système de pilotage par la performance. A ce titre, les présidents des conseils généraux étudiés ont, tous, été soit à l'initiative de la démarche, soit associés au projet et relayeur de la démarche. La différence pourrait, par contre, provenir de la participation des directeurs généraux des services départementaux

\footnotetext{
${ }^{8}$ Dans certains collectivités, le terme est différent (le « tableau de bord annuel des activités » dans le CG5), mais la structure documentaire permet d'apprécier le périmètre concerné par la démarche de performance et le reporting d'information sur cette performance.

${ }^{9}$ En synthèse, voir Bartoli (1997).
} 
(DGS) et des directeurs opérationnels à la démarche (avant la validation du cadre général), observée dans les quatre premiers $C G$. Ainsi dans le $\mathrm{CG} \mathrm{n}{ }^{\circ} 5$, où le périmètre de la démarche est plus restreint, nous pourrions envisager que l'absence des directeurs opérationnels dans les premières réunions (seul le DGS était présent) a limité la généralisation de la démarche. L'étude des modalités de mise en œuvre nous éclaire sur ces éléments.

Concernant les éléments organisationnels, puis méthodologiques, des modalités mises en oeuvre, nous avons pu observer des différences significatives par rapport à la mise en œuvre de la LOLF. Ces différences tiennent à l'implication des élus et des fonctionnaires et la création d'interactions entre les différents acteurs, la mise en œuvre d'un plan pluriannuel d'implémentation et l'adaptation de la démarche aux caractéristiques des structures concernées, et à la tentative d'enracinement progressif du changement culturel induit. En effet, au sein de chacun des conseils généraux, un portage d'élus locaux et directeurs administratifs est présent. Excepté pour le CG 5, où seul le DGS participait aux premières réunions, un soutien fort des élus et des cadres administratifs est recherché dans les démarches de performance publique locale. Ce soutien prend la forme d'interactions formalisées au travers de comités de pilotage $\left(\mathrm{CG} \mathrm{n}^{\circ} 1,2,4\right)$, comités de projets $\left(\mathrm{CG} \mathrm{n}^{\circ} 2,3,4\right)$, de référents dans les directions $\left(\mathrm{CG} \mathrm{n}^{\circ} 2,3,5\right)$.

D'un point de vue méthodologique, la mise en œuvre est identique dans chaque CG : un plan pluriannuel d'implémentation est fixé par le comité en charge de la démarche, et cette dernière est mise en place de manière adaptée dans les directions. Dans le CG $\mathrm{n}^{\circ} 1$, trois années de déploiement sont planifiées, dans les autres CG deux années étaient annoncées mais les délais ne sont pas toujours respectés afin de s'insérer dans les pratiques de fonctionnement des directions opérationnelles. Concrètement, le groupe projet ou comité de pilotage à chaque fois permis d'obtenir des outils négociés, des indicateurs acceptés par les acteurs locaux. En effet, la dernière spécificité observée concerne la recherche d'enracinement progressif du changement culturel induit. Si la contingence des démarches aux contextes locaux et l'approche participative retenue (les comités de pilotage ou de projets transversaux ont animé de nombreuses réunions avec les acteurs des différents services pour impliquer un maximum d'agents) prennent un temps certain, celui-ci est accepté par les équipes de direction afin de prendre en compte les changements culturels et techniques induits par les DPL. Nos observations ne permettent pas de dire si ce point est validé car l'étude nécessiterait un recul sur plusieurs années, mais la volonté des dirigeants reste affichée.

Ces modalités s'opposent à celles de la LOLF dans les administrations, où l'implication des directeurs n'était pas prioritaire, le calendrier de mise en œuvre étant trop serré pour intégrer de la participation et les outils étant imposés (tableaux de reporting et indicateurs déjà formalisés).

Si des divergences apparaissent au niveau du périmètre et des modalités de mise en oeuvre, d'autres existent entre les objectifs des démarches locales de performance et de la LOLF.

\subsubsection{Les objectifs poursuivis à travers les démarches et les indicateurs associés}

A l'instar du point précédent sur le périmètre et les modalités, nous nous appuyons sur les documents et entretiens, et nous restituons en italique les écrits communs à différents supports internes repris par les responsables lors des entretiens. 
Les cinq collectivités étudiées affichent ainsi, comme objectif attendu de leur démarche de performance, la diminution des coûts de fonctionnement (repris par les DGS lors des entretiens). Cependant, les démarches diffèrent sur les autres buts poursuivis. Dans le $\mathrm{CG} \mathrm{n}^{\circ} 1$, les objectifs sont de quatre ordres : «développer une culture de résultat avec une nouvelle gouvernance (Améliorer la lisibilité des politiques publiques, des priorités, afficher des objectifs et des stratégies de programme, évaluer et rendre compte de l'action), améliorer l'offre des services publics en lien avec les besoins du territoire, garantir une totale sécurité juridique et améliorer la maîtrise des coûts » (sources : site intranet du CG, rapport de performance, entretien DGS). Pour le CG $\mathrm{n}^{\circ} 2$, la démarche de performance s'insère dans «le cadre des plans de modernisation de l'administration et la démarche de développement durable », et elle vise à "améliorer la qualité de service tout en conservant une efficacité et une efficience dans les actions, à consolider l'attractivité territoriale, à améliorer la lisibilité des politiques publiques et la reddition des comptes » (sources : site intranet du CG, rapport de performance, entretien DGS). Le $\mathrm{CG}^{\circ} 3$ reprend certains des objectifs du CG ${ }^{\circ} 2$ dans sa démarche de performance visant «une meilleure performance énergétique, économique et sociale pour la collectivité » (sources : site intranet du CG, rapport de performance). Les objectifs des $\mathrm{CG} \mathrm{n}^{\circ} 4$ et $\mathrm{n}^{\circ} 5$ portent, eux, sur l'amélioration de la maîtrise des coûts, la qualité environnementale et l'amélioration de la lisibilité des politiques publiques pour les citoyens (sources : rapports de performance, le tableau de bord annuel des activités, les entretiens DGS). La portée des buts est moins large que dans les deux premiers $\mathrm{CG}$, intégrant les notions de territoire et de sécurité. Cependant, nous retrouvons dans ces libellés la diversité des objectifs pouvant être poursuivi à travers les démarches de performance publique locale.

Si nous comparons avec le niveau national, la LOLF est un moyen pour les administrations d'atteindre trois objectifs principaux : rendre les documents budgétaires plus lisibles (transparence accrue pour le débat public), une allocation des crédits améliorée par les missions et objectifs fixés, une plus grande responsabilisation des gestionnaires publics engagés sur les objectifs. Ainsi, les principes LOLFiques n'intègrent pas la notion de territoire, de développement durable, ni de parties prenantes (si ce n'est la prise en compte des attentes d'information du citoyen).

Cependant, la diversité des objectifs affichés par les collectivités peut être nuancée lors de la mise en œuvre des démarches de performance avec les indicateurs mobilisés. En effet, afin de «vérifier » la portée des objectifs affichés, nous avons recherché, dans les documents de travail, les concepts mobilisés et faisant l'objet d'une mesure. Naturellement, des décalages apparaissent entre les intentions annoncées et les mises en œuvre. Mais l'intérêt se situe principalement dans le degré d'expérimentation par rapport au modèle de la LOLF.

Si traditionnellement sont distingués les indicateurs de performance qui mesurent l'atteinte des objectifs stratégiques et les indicateurs de pilotage qui mesurent l'évolution des facteurs clés permettant la gestion courante, la LOLF propose elle une typologie en indicateurs d'efficience, d'efficacité et de qualité pouvant servir au pilotage ou à la mesure de performance. En effet, la LOLF propose trois catégories de concepts faisant l'objet de trois familles d'indicateurs : l'efficience de la gestion pour le contribuable, la qualité de service pour l'usager, l'efficacité pour les citoyens. 
En parallèle, le tableau qui suit présente les objectifs affichés, les concepts et indicateurs mesurés dans chacun des cinq conseils généraux.

Tableau 3 : Les objectifs, concepts et indicateurs retenus dans les démarches de performance des CG

\begin{tabular}{|c|c|c|c|c|c|}
\hline & CG1 & CG2 & CG3 & CG4 & CG5 \\
\hline $\begin{array}{l}\text { Les objectifs } \\
\text { affichés } \\
\text { (améliorer...) }\end{array}$ & $\begin{array}{l}\text { Culture du } \\
\text { résultat, maîtrise } \\
\text { des coûts, } \\
\text { gouvernance, } \\
\text { qualité de l'offre } \\
\text { des services } \\
\text { publics, sécurité } \\
\text { juridique }\end{array}$ & $\begin{array}{l}\text { Modernisation, } \\
\text { gouvernance, } \\
\text { développement } \\
\text { durable, } \\
\text { attractivité, } \\
\text { qualité service }\end{array}$ & $\begin{array}{l}\text { Performance } \\
\text { énergétique, } \\
\text { performances } \\
\text { économique } \\
\text { et sociale }\end{array}$ & $\begin{array}{l}\text { Culture } \\
\text { du résultat, } \\
\text { performances } \\
\text { environnementales, } \\
\text { lisibilité } \\
\text { des comptes } \\
\text { et de actions }\end{array}$ & $\begin{array}{l}\text { Culture } \\
\text { du résultat, } \\
\text { performances } \\
\text { environnementales, } \\
\text { lisibilité } \\
\text { des comptes } \\
\text { et de actions }\end{array}$ \\
\hline $\begin{array}{l}\text { Les concepts } \\
\text { mesurés } \\
\text { (et les angles } \\
\text { d'analyse) }\end{array}$ & $\begin{array}{l}\text { Efficience } \\
\text { (contribuables), } \\
\text { Qualité réalisée } \\
\text { et perçue } \\
\text { (usagers et } \\
\text { agents), } \\
\text { efficacité et } \\
\text { pertinence } \\
\text { (citoyens), } \\
\text { impacts sur le } \\
\text { territoire } \\
\text { (élus) }\end{array}$ & $\begin{array}{l}\text { Efficience, } \\
\text { efficacité, } \\
\text { Qualité réalisée, } \\
\text { impacts } \\
\text { sur le territoire }\end{array}$ & $\begin{array}{l}\text { Efficience, } \\
\text { efficacité, } \\
\text { impacts sur } \\
\text { le territoire }\end{array}$ & $\begin{array}{l}\text { Efficience, } \\
\text { efficacité, } \\
\text { pertinence } \\
\text { des actions } \\
\text { impacts sur } \\
\text { le territoire, } \\
\text { qualité réalisée } \\
\text { et perçue de l'offre }\end{array}$ & $\begin{array}{l}\text { Efficience, impacts } \\
\text { sur le territoire }\end{array}$ \\
\hline $\begin{array}{l}\text { Les } \\
\text { indicateurs } \\
\text { mobilisés } \\
\text { dans les } \\
\text { démarches } \\
\text { de } \\
\text { performance }\end{array}$ & $\begin{array}{l}\text { Indicateurs } \\
\text { financiers } \\
\text { (Efficience), } \\
\text { indicateurs } \\
\text { de suivi d'action } \\
\text { (efficacité et } \\
\text { qualité réalisée } \\
\text { - les délais), } \\
\text { indicateurs de } \\
\text { pertinence, } \\
\text { indicateurs } \\
\text { de contexte } \\
\text { (impacts sur } \\
\text { le territoire), } \\
\text { indicateurs sur } \\
\text { le climat social } \\
\text { (les agents) }\end{array}$ & $\begin{array}{l}\text { Indicateurs } \\
\text { financiers, } \\
\text { indicateurs de } \\
\text { suivi d'action } \\
\text { (efficacité et } \\
\text { qualité réalisée } \\
\text { - les délais), } \\
\text { indicateurs } \\
\text { de pertinence, } \\
\text { indicateurs } \\
\text { de contexte } \\
\text { (impacts sur } \\
\text { le territoire) }\end{array}$ & $\begin{array}{l}\text { Indicateurs } \\
\text { financiers } \\
\text { (Efficience), } \\
\text { indicateurs } \\
\text { de suivi } \\
\text { d'action } \\
\text { (efficacité), } \\
\text { indicateurs } \\
\text { de contexte }\end{array}$ & $\begin{array}{l}\text { Indicateurs } \\
\text { financiers } \\
\text { (Efficience), } \\
\text { indicateurs de } \\
\text { suivi d'action, } \\
\text { indicateurs } \\
\text { de pertinence, } \\
\text { indicateurs } \\
\text { de contexte }\end{array}$ & $\begin{array}{l}\text { Indicateurs } \\
\text { financiers } \\
\text { (Efficience), } \\
\text { indicateurs } \\
\text { de suivi d'action }\end{array}$ \\
\hline
\end{tabular}

Si les soubassements du modèle classique de performance publique outputs-outcomes (Gibert, 2001) apparaissent à travers les mesures de l'efficience des actions (consommation budgétaire des programmes), l'efficacité (degré d'atteinte des objectifs des programmes) et des impacts sur le territoire, l'intérêt porté à différents angles d'analyse pour les mesures dépasse le cadre de la LOLF dans trois CG et s'apparente à une approche adaptée du balanced scorecard. En effet, les collectivités étudiées ont rajouté les attentes de l'élu local, se différenciant des autres angles d'analyse par l'importance accordée aux impacts des politiques publiques sur le territoire de compétence. De plus, 
la pertinence (les ressources mobilisées par rapport aux projets politiques) et les impacts sur le territoire sont recherchés dans la majorité des CG. Une collectivité mobilise aussi des indicateurs sur le climat social.

Les collectivités entrées dans une démarche de performance ont ainsi élargi l'évaluation de la performance à des notions dépassant les aspects économiques et financiers. En cela, elles ne font pas de choix entre une performance orientée input, output ou outcome, mais tentent d'appréhender chaque élément. Cependant, nous avons pu noter, que dans les directions, plus de la moitié des indicateurs préexistaient à la démarche. Seuls quelques indicateurs ont été créés pour la circonstance, ces derniers étant ceux qui aujourd'hui posent le plus de problèmes en termes de collectes car les sous-directions n'en voient pas toujours l'intérêt et, bien souvent, n'en maîtrisent pas le calcul.

Dans les deux premiers CG, où l'affichage des mesures retenues pour apprécier la performance est le plus large, nous avons pu lister dans les documents de travail des indicateurs proches de ceux préconisés par les auteurs du BSC. Ainsi, dans le CG n ${ }^{\circ}$, la synthèse des indicateurs présents dans les tableaux de bord mensuels ou trimestriels des programmes d'action s'apparente à une adaptation du BSC (tableau n $\left.{ }^{\circ} 4\right)$. Les fiches programmes sont issues du travail pendant quatre mois d'un groupe transversal de responsables de services et directeurs ayant formalisé une maquette de tableau de bord « idéal ». La maquette de tableau de bord obtenue, dans le tableau ci-dessous, illustre les divers axes d'analyse retenus dans la démarche de pilotage de la performance. La difficulté à définir des indicateurs est contournée par l'élaboration collective (groupe de travail avec un représentant de chaque direction) de cette maquette, proposée à chaque direction. Cependant, le renseignement des items est partiel dans les deux axes situés dans le bas du tableau. Par ailleurs, la part des indicateurs rétrospectifs et prospectifs, théoriquement équilibrée dans le BSC, n'est en pratique que rarement observée. Nous pouvons cependant considérer que les efforts menés pour renseigner certains items dans l'axe apprentissage correspondent à des données prospectives et vont influencer l'évolution des autres axes. Cette nuance ne cache pas une autre limite liée à l'élaboration. En effet, suivant les recommandations de la construction du BSC, un groupe de travail transversal a défini les axes et sélectionné des indicateurs afin d'obtenir un alignement stratégique des différents services et d'obtenir un consensus des acteurs. Cependant, le cadre de cette maquette a vocation à être adapté car si la moitié des directions et services remplie une majorité d'items, l'autre moitié concerne des directions où une minorité d'indicateurs sont renseignés. Nous constatons donc, dans ce CG, une référence explicite au BSC pour représenter les indicateurs de performance. Mais si la structuration et l'élaboration en sont inspirées, les caractéristiques de causalité et d'équilibre dans les indicateurs ne sont pas présentes. 
Tableau 4 : Maquette du tableau de suivi de la performance dans le $\mathrm{CG} \mathrm{n}^{\circ} 1$

\begin{tabular}{|c|c|}
\hline \multicolumn{2}{|l|}{ Tableau de bord pour la période : } \\
\hline \multicolumn{2}{|l|}{ Eléments de contexte: } \\
\hline Axe bénéficiaire/usager/citoyen & Axe financier \\
\hline $\begin{array}{l}\text { Indicateurs de résultats } \\
\text { socioéconomiques } \\
\text { (taux de chômage, de pollution...) } \\
\text { Indicateurs de qualité de service } \\
\text { (enquête par mission ou par programme) }\end{array}$ & $\begin{array}{l}\text { Niveau de réalisation budgétaire } \\
\text { (comparaison des prévisions et des réalisations par mission); } \\
\text { Indicateurs de coût total des missions et programmes } \\
\text { avec les dépenses directes, les frais personnel, } \\
\text { les postes importants; } \\
\text { Indicateurs significatifs de coût par prestation }\end{array}$ \\
\hline Axe processus internes & Axe apprentissage organisationnel \\
\hline $\begin{array}{l}\text { Délai de traitement d'une commande } \\
\text { ou demande d'aide (aide individuelle, } \\
\text { subvention, délai d'instruction } \\
\text { et de paiement) } \\
\text { Délai de production d'une délibération } \\
\text { (délai de production des rapports, } \\
\text { délai d'expédition, délai de publication) } \\
\text { Ecarts de réalisation des projets } \\
\text { d'investissement (écart entre prévision } \\
\text { et délais contractuels et la réalisation) } \\
\text { Niveau de dématérialisation des } \\
\text { processus de réponse aux clients/usagers } \\
\text { (réponse en ligne, dématérialisation } \\
\text { des appels d'offres, formulaires } \\
\text { de demande d'aide) }\end{array}$ & $\begin{array}{l}\text { Motivation : } \\
\mathrm{Nb} \text { de jours d'arrêt maladie, nb d'agents concernés } \\
\mathrm{Nb} \text { de sollicitations signalées au service du personnel } \\
\text { Compétences : } \\
\text { Niveau moyen de compétences des agents / niveau moyen } \\
\text { des compétences requises avec les entretiens d'évaluation } \\
\text { Nb de jours de formation et nb d'agents concernés } \\
\text { Projets de modernisation : } \\
\text { Nb de projet de services en cours } \\
\text { Etat d'avancement des projets de modernisation } \\
\text { Niveau d'appropriation des démarches de modernisation } \\
\text { engagées }\end{array}$ \\
\hline
\end{tabular}

Source : annexe aux documents internes sur la démarche de performance du $C G$.

Dans le $\mathrm{CG} \mathrm{n}^{\circ} 2$, les rapports de performance sont structurés de manière quasi identique. Les directions font état des programmes réalisés, ou en cours de réalisation, en indiquant les éléments sur les quatre axes, présentés en quatre parties qui suivent les données de contexte. Le fait d'avoir un logiciel de géo-référencement avec certaines données alimentant un SI géographique permet au CG de renseigner des éléments de contexte précis sur le territoire, intégrant le développement durable. Concernant l'élaboration, les mêmes caractéristiques se retrouvent aussi. Un groupe de travail composé de responsables de chaque direction s'est réuni durant quatre mois pour élaborer une maquette de tableau de bord équilibré, puis les services se sont vus proposer les documents. Les absences de causalité entre les quatre parties du tableau et d'indicateurs prospectifs sont également observées dans les documents.

Concernant les trois autres collectivités, les indicateurs mobilisés appartiennent aux axes d'analyse de la performance financière (indicateurs sur la consommation budgétaire, le coût des programmes) et des processus internes (indicateurs de délais), avec des indicateurs sur la satisfaction des bénéficiaires et des habitants (orientation citoyen) associés à des indicateurs de contexte économique. Nous retrouvons ici l'approche de la performance exposée dans la LOLF, qui distingue les indicateurs de contexte, de résultats (axes bénéficiaire et citoyen), d'efficience et d'efficacité (axe financier). 
Ainsi, dans les CG 3 et 4, les fiches « Programme » comprennent la mission de rattachement, les éléments d'environnement (indicateurs socio-économiques) et les attentes des parties prenantes, avec des indicateurs d'efficacité et de qualité en terme de délais, et des indicateurs d'efficience (les crédits d'intervention et les ressources mobilisées). Les informations du « Programme » sont parfois détaillées par actions, comprises au sein du programme, et présentées de manière proche aux recommandations de la LOLF. Ainsi, dans le rapport annuel du $\mathrm{CG} \mathrm{n}{ }^{\circ} 4$, les fiches Programmes sont synthétisées et mentionnent pour chaque action les éléments indiqués dans le tableau n ${ }^{\circ} 5$.

Tableau $\mathbf{n}^{\circ} \mathbf{5}$ : Extraits de deux actions appartenant au programme Jeunesse dans le CG $n^{\circ} 4$.

\begin{tabular}{|c|c|c|c|c|c|c|}
\hline $\begin{array}{c}\text { Cible } \\
\text { quantifiée }\end{array}$ & $\begin{array}{c}\text { Objectif } \\
\text { opérationnel }\end{array}$ & Dispositif & Obligation & $\begin{array}{l}\text { Coût } \\
\text { direct } \\
\text { au BP }\end{array}$ & Intervention & Capacité d'accueil \\
\hline $\begin{array}{l}\text { Écoliers } \\
\text { en difficulté } \\
\text { de lecture }\end{array}$ & $\begin{array}{l}\text { Appuyer les } \\
\text { apprentissages }\end{array}$ & $\begin{array}{l}\text { Plan d'aide } \\
\text { à la lecture } \\
\text { (PAL), } \\
\text { créé en } 1989\end{array}$ & Non & 607000 & $\begin{array}{l}\text { En directe } \\
\text { avec les } \\
\text { communes }\end{array}$ & 2900 écoliers \\
\hline $\begin{array}{c}\text { Elèves de } \\
6^{\mathrm{e}} \text { et } 5^{\mathrm{e}} \text { en } \\
\text { difficulté, } \\
\text { soit } 7 \% \text { de } \\
\text { l'effectif, }^{10800 .}\end{array}$ & $\begin{array}{c}\text { Appuyer les } \\
\text { apprentissages }\end{array}$ & $\begin{array}{c}\text { Atelier } \\
\text { pédagogique, } \\
\text { créé en } 1995\end{array}$ & Non & 954500 & $\begin{array}{l}\text { Intervention } \\
\text { directe }\end{array}$ & $\begin{array}{c}\text { Capacité } \\
\text { théorique : } 7200 \\
\text { collégiens }\end{array}$ \\
\hline
\end{tabular}

Les indicateurs de chaque action font références à plusieurs acteurs, à savoir le citoyen (impact de la politique suivie, image de la collectivité et des élus), le contribuable (participation à la politique, efficience organisationnelle, économie de ressources pour les impôts), l'usager (qualité et valeur ajoutée des services délivrés) et les agents ou la tutelle (lier les processus et l'organisation, standardiser le service par des procédures).

Cependant, chaque action n'est pas renseignée en totalité. Ainsi, une évaluatrice du CG 4 explique que "pour la fixation des objectifs, il faut reconnaître que certains services administratifs ne les ont pas encore définis. Ainsi, ce n'est pas pertinent dans mon domaine de l'évaluation. Je vois mal ce que je pourrais mettre comme indicateur pour un agent $d u$ service, car parfois on a des retards et je ne peux pas imputer les difficultés à la personne car ce sont des contraintes extérieures qui expliquent le retard. ».

Ainsi, les indicateurs mobilisés dans les démarches de performance observées s'inspirent du BSC dans les deux premiers conseils généraux, et pour les trois autres une 
filiation s'observe avec la LOLF. Si l'influence de la BSC sur les systèmes de gestion reste faible du fait des indicateurs non remplis et l'absence de causalité ou hiérarchie entre les axes d'analyse, l'influence des principes Lolfiens apparaît plus forte à travers les instruments mobilisés.

\subsubsection{Les instruments et pratiques mobilisés dans les démarches}

De la même façon que les indicateurs utilisés, les outils mis en œuvre dans les démarches de performance correspondent en grande partie à ceux présentés dans le Guide méthodologique de la LOLF à destination des administrations (Coll., 2004, op.cit. et 2007).

\begin{tabular}{|c|c|c|c|}
\hline $\begin{array}{l}\text { Public touché } \\
\text { (nb) et coût }\end{array}$ & $\begin{array}{l}\text { Lisibilité } \\
\text { (usagers } \\
\text { et prof.) }\end{array}$ & Valeur ajoutée & Propositions \\
\hline $\begin{array}{l}2700 \text { écoliers, } \\
225 € / \text { écolier }\end{array}$ & moyenne & $\begin{array}{l}\text { Site PAL : } 300 \\
\text { connexions/j; } \\
\text { le PAL intervint } \\
\text { à l'âge où l'on } \\
\text { peut remédier } \\
\text { effectivement } \\
\text { aux difficultés } \\
\text { avec un appui } \\
\text { assez léger. } \\
\text { Programme } \\
\text { contractualisé } \\
\text { avec les } \\
\text { communes }\end{array}$ & $\begin{array}{c}\text { faible lisibilité } \\
\text { du CG auprès } \\
\text { des parents. } \\
\text { Compte tenu } \\
\text { des actions } \\
\text { du CG en } \\
\text { direction des } \\
\text { collèges on peut } \\
\text { s'interroger sur } \\
\text { la pertinence } \\
\text { de cette action } \\
\text { pédagogique } \\
\text { sous } \\
\text { compétence } \\
\text { municipale }\end{array}$ \\
\hline $\begin{array}{c}7200, \text { soit } 132 \\
\text { €/collégiens }\end{array}$ & $\begin{array}{c}\text { Faible, } \\
\text { manque de } \\
\text { communication } \\
\text { auprès } \\
\text { des parents }\end{array}$ & $\begin{array}{c}\text { Après } \\
\text { évaluation, } \\
\text { réduction du } \\
\text { nb de thèmes } \\
\text { d'ateliers. }\end{array}$ & $\begin{array}{l}\text { A maintenir, et } \\
\text { nécessité d'une } \\
\text { communication } \\
\text { directe auprès } \\
\text { des parents }\end{array}$ \\
\hline
\end{tabular}

Dans ce guide, les instruments proposés concernent la présentation des budgets en missions, programmes et actions (MPA), la définition d'objectifs et d'indicateurs matérialisés dans des projets annuels de performance (PAP) et des rapports annuels de performance (RAP) pour comparer les deux documents, le suivi des objectifs intermédiaires déclinés des objectifs stratégiques dans des tableaux de bord (TB). De plus, sont exposées des pratiques de déconcentration, de responsabilisation et d'évaluation : une délégation de la gestion des programmes aux responsables, un dialogue de gestion pour les responsables de services, un entretien annuel d'évaluation pour chaque agent fixant des objectifs personnels et évaluant leur contribution aux résultats.

Nous avons pu observer, dans chacun des CG, la mise en place d'une déclinaison des politiques publiques en MPA associée à une présentation budgétaire. Un compte rendu de la performance de chaque programme est présenté aux élus locaux à travers un document proche du RAP, ce dernier sert essentiellement aux débats d'orientation budgétaire. Par ailleurs, dans les cinq collectivités étudiées, l'instauration d'un plan annuel de missions d'audits et/ou d'évaluation de politiques publiques afin d'apprécier les effets des actions menées sur le territoire est présent dans les CG n², 3, 4, la collectivité $n^{\circ} 1$ assurant des audits et évaluations mais sans les planifier annuellement. En outre, la plupart des collectivités ont développé un système d'information géographique (SIG) pour faciliter les évaluations et les répondre aux informations attendues par les élus sur leur territoire. 
Tableau 6 : Synthèse des instruments et pratiques issus des démarches observées

\begin{tabular}{|c|c|c|c|c|c|}
\hline & CG $n^{\circ} 1$ & $\mathrm{CG} \mathbf{n}^{\circ} 2$ & CG n³ & CG n ${ }^{\circ} 4$ & CG n5 \\
\hline $\begin{array}{l}\text { Les } \\
\text { instruments } \\
\text { utilisés }\end{array}$ & $\begin{array}{l}\text { Budget en MPA, } \\
\text { début de comptabilité } \\
\text { par activités, } \\
\text { TB équilibré et RAP, } \\
\text { évaluations de } \\
\text { politiques }\end{array}$ & $\begin{array}{l}\text { Budget en } \\
\text { MPA, } \\
\text { TB équilibré, } \\
\text { SIG, RAP, } \\
\text { évaluations de } \\
\text { politiques, }\end{array}$ & $\begin{array}{l}\text { Budget en } \\
\text { MPA, } \\
\text { SIG, audits et } \\
\text { évaluations de } \\
\text { politiques }\end{array}$ & $\begin{array}{l}\text { Budget en MPA, } \\
\text { SIG, RAP, } \\
\text { audits et } \\
\text { évaluations de } \\
\text { politiques }\end{array}$ & $\begin{array}{l}\text { Budget } \\
\text { en MPA, } \\
\text { SIG }\end{array}$ \\
\hline $\begin{array}{l}\text { Les } \\
\text { pratiques }\end{array}$ & $\begin{array}{l}\text { démarche } \\
\text { suivant la LOLF } \\
\text { (déconcentration, } \\
\text { responsabilisation et } \\
\text { évaluation) }\end{array}$ & $\begin{array}{l}\text { démarche } \\
\text { suivant } \\
\text { la LOLF, } \\
\text { modernisation } \\
\text { des SI }\end{array}$ & $\begin{array}{l}\text { modernisation } \\
\text { des SI }\end{array}$ & $\begin{array}{l}\text { démarche de } \\
\text { responsabilisation } \\
\text { collective, }\end{array}$ & $\begin{array}{l}\text { modernisation } \\
\text { des SI }\end{array}$ \\
\hline
\end{tabular}

Dans le CG ${ }^{\circ}{ }^{1}$, il existe des fiches annuelles de performance, sur la base des rapports annuels de performance développés dans la LOLF, qui se présentent sous la forme de tableaux de bord équilibrés. En s'appuyant sur le début d'une comptabilité par activités (coût par programmes et parfois par actions), elles complètent la budgétisation par MPA. Parallèlement, pour obtenir une « gestion par les résultats », une démarche de responsabilisation du personnel a été initiée, qui comprend des primes individuelles et collectives attribuées selon l'atteinte des objectifs fixés, lors des entretiens annuels d'évaluation, sur la base de la stratégie votée. La déclinaison des objectifs dans chaque service et la part variable de rémunération sont censées motiver le personnel dans l'atteinte des résultats attendus par les élus sur chaque mission ou programme. Les mêmes outils sont utilisés dans les $\mathrm{CG} \mathrm{n}{ }^{\circ} 2$ et 4 , hormis la comptabilité par activités et le principe de prime individuelle qui caractérisent seulement le $\mathrm{CG} \mathrm{n}^{\circ} 1$.

Le $\mathrm{CG}^{\circ} 4$ a lui développé des rapports annuels de performance comme le propose la LOLF et a assorti l'obtention des résultats à des primes collectives. Pour assister la démarche d'évaluation des politiques visant à identifier les impacts des grands programmes menés, un système d'information géographique (SIG) a été élaboré. Ce SIG est un outil que l'on peut également observer dans les $\mathrm{CG} \mathrm{n}^{\circ} 2,3$ et 5 . Il est par contre à l'état de projet dans le premier CG. Généralement associé à un logiciel tel que MapInfo, il permet de géo-référencer les aides de la collectivité et les effets directs des actions sur le territoire, ce qui aide les évaluateurs dans leurs missions et permet de constituer des rapports complets à destination des élus et/ ou de la population sur les politiques publiques.

Ces différents outils permettent, au total, de répondre à la mesure des concepts retenus dans les démarches locales de performance publique. Nous nous apercevons ainsi que ces démarches sont transversales, en affectant le système comptable et budgétaire (budgétisation en MPA, introduction comptabilité analytique), le système de reporting d'informations (SI géographique, recherche des impacts et des contextes des politiques publiques), le système managerial (responsabilisation et primes sur objectifs), mais peu, ou pas, le système organisationnel (les organigrammes fonctionnels ne sont pas modifiés en fonction des responsabilités transversales, ni création de centres de responsabilités, hormis dans le CG $\mathrm{n}^{\circ} 1$ où les principes de la LOLF sont quasi-appliqués). Le souhait des quatre directeurs 
généraux (parmi les cinq conseils généraux) de ne pas apporter de changements dans les organigrammes, et donc dans les services, s'explique principalement par la volonté d'adapter la démarche de performance au fonctionnement existant. Lorsque nous avons émis le risque de caller de nouveaux principes de fonctionnement dans un cadre non adapté, les réponses ont porté sur les modifications des caractéristiques de la démarche à la collectivité. Aussi, les contenus que nous exposons ne sont pas figés et peuvent être amendés pour mieux correspondre à des souhaits internes.

Pour synthétiser, ces différents résultats montrent une vue nuancée des démarches de performance publique locale. Malgré la diversité des objectifs annoncés, les outils et indicateurs mobilisés sont proches de ceux recommandés par la LOLF et limitent donc l'atteinte des objectifs ou des ambitions affichées. Cette caractérisation des démarches locales de performance au regard de notre grille de lecture inspirée de celle mise en œuvre par la LOLF, nous permet ainsi de répondre au final à notre problématique de recherche.

\section{Des éléments contingents et spécifiques, gages de réussite des démarches locales de performance}

Les observations dans certains conseils généraux nous renseignent sur les éléments constitutifs des premières démarches volontaires en France de performance publique locale. Ces résultats mettent en évidence, d'un côté, un certain mimétisme avec la LOLF, sur des éléments caractéristiques, et de l'autre, quelques spécificités, notamment en termes de modalités de mise en œuvre et d'intérêt à mobiliser une approche globale de la performance.

\subsection{Une diversité limitée par rapport à la LOLF en terme d'outils, mais importante en terme de modalités}

Il ressort de cette étude que le contenu global des démarches de performance locale est proche de celui présenté dans la LOLF. Ce rapprochement des démarches volontaires des collectivités auprès d'un cadre institutionnalisé peut s'expliquer par le souhait d'avoir un référentiel, qui est ensuite adapté à la finalité de développement territorial. Cet argument est avancé par les quatre DGS des CG n $1,2,3,4$, alors que le DGS du CG n5 n'a «pas essayé de calquer la démarche LOLF à la collectivité», mais a créé des "groupes de réflexion sur l'opportunité et le contenu d'une démarche de performance pour obtenir une démarche ad $h o c »$. Nous pouvons également expliquer cette proximité avec la LOLF pour son intérêt intrinsèque. Si les risques d'une démarche de performance locale concernent la fragilisation du pouvoir local en mettant en évidence des écarts entre les objectifs annoncés et les résultats obtenus, pour Huteau (2008), des bénéfices existent car « une caractéristique de la LOLF est de redonner aux élus locaux leur réel pouvoir d'orientation de l'action publique ».

Ce mimétisme confirmerait l'isomorphisme normatif et l'isomorphisme mimétique étudiés par les théoriciens néo-institutionnels, dans le champ de la performance publique locale. En effet, d'une part, la légitimité de la démarche de performance fait référence à des éléments édictés par les milieux professionnel, dont la formation est un vecteur important (Scott, 1995), et, d'autre part, en situation d'incertitude, il semble légitime de copier ce que font les autres (Dambrin et al., 2005). Le recours à des éléments de la LOLF peut s'expli- 
quer par les apports techniques adaptés à des exigences que doivent gérer les responsables locaux et par des principes adaptés aux enjeux auxquels font face des collectivités locales. Ainsi, Maltais et Mazouz (2004) soulignent que la logique de performance associée à la LOLF ne remet pas en cause directement le rôle de l'Etat, mais questionne son organisation et son fonctionnement.

Néanmoins, nous avons observé des modalités de mise en œuvre très différentes de celles pratiquées lors de l'implémentation de la LOLF dans les administrations. En effet, des différences ont été mises en évidence concernant des éléments à la fois organisationnels et méthodologique. Ces derniers permettent d'opposer les modalités des démarches de performance locale à celles d'une démarche nationale, à travers la mise en œuvre de la LOLF. Précisément, les points de divergences mis en exergue portent sur l'implication politique des élus et des fonctionnaires, la création d'interactions entre les différents acteurs, l'adaptation de la démarche aux caractéristiques des structures concernées et la création puis l'enracinement progressif du changement culturel induit. Ces éléments confirment les résultats de précédentes recherches portant sur l'implémentation des réformes dans les collectivités (Carassus et al., 2010), mais apportent également des points d'analyse. Ces différences constituent autant de facteurs de succès pouvant être mobilisés pour d'autres projets managériaux ou d'autres démarches dites de modernisation du fonctionnement des collectivités.

Parallèlement, pour atteindre leurs objectifs affichés dans les démarches, les collectivités visitées ont généré de nouveaux outils prospectifs, à travers un observatoire fiscal ou un SIG, et d'évaluation, à travers des indicateurs dépassant le cadre de la LOLF avec une approche sociale sur le capital humain et des tentatives d'approche sociétale avec le développement durable du territoire. Les résultats correspondent donc à un certain mimétisme instrumental et de pratiques, parallèlement à de relatives innovations dans les modalités de mise en œuvre et les enjeux fixés. On aboutit au total à des « innovations mimétiques », où coexistent un noyau commun (des outils de gestion publique) et, à la périphérie, des éléments contingents (les modalités, enjeux..). Le contenu d'une démarche de performance d'une collectivité, résultant a priori des choix des dirigeants locaux, semble alors être affecté à la fois par des effets de contagion (voisinage, mimétisme), de rattrapage, et de migration (rapprochement d'une « structure innovante »), qui vont diminuer les disparités. En l'espèce, les DPL ne sont ni des approches calquées de la LOLF ni de la BSC, ni du CAF, elles empruntent à chacune des caractéristiques. Cette adaptation ne constitue pas une surprise en soi tant la contingence est de mise en management public. Pour autant, la mise en évidence de traits convergents (les instruments) parallèlement à la recherche de modalités spécifiques participatives représente un résultat émergent.

Les objectifs poursuivis dans les cinq CG illustrent ainsi le caractère multiple des contenus de la performance publique locale, qui dépassent les objectifs de la LOLF principalement orientés vers l'intérieur de l'organisation. Déjà, la conception de la performance retenue dans la LOLF comprenait différents niveaux (stratégique, tactique, opérationnel), différentes périodes (court terme, moyen terme, long terme), et différentes dimensions (économique, financière, sociale), ce qui constitue une vision large de la performance, mais les collectivités semblent vouloir aller plus loin. Outre la réduction des coûts de fonctionnement qui est présente dans chaque démarche de performance des conseils généraux, nous notons un intérêt pour l'approche $\mathrm{BSC}$, comme l'enquête de Chauvey (2005), avec des adaptations sur l'axe usager-bénéficiaire intégrant les effets des politiques sur le territoire. Cependant, pour élaborer le tableau de bord prospectif, les auteurs 
recommandent une approche top-down pour favoriser l'alignement stratégique des directions, alors que ce caractère dirigiste s'oppose à la volonté partenariale de l'élaboration des mesures dans chaque axe du tableau (Gibert, 2002). Par ailleurs, les quatre axes de performance du BSC sont considérés comme interreliés (Kaplan, 2010). Or, la représentation de la performance sous forme de relations réciproques complexifie la prise de décision dans les collectivités, puisque la hiérarchie des parties prenantes n'y est pas affirmée ${ }^{10}$.

Malgré ces limites théoriques et pratiques, des collectivités visitées adaptent l'approche BSC, notamment pour les indicateurs de l'axe «client» remplacés par quelques ratios relatifs aux usagers, même si les principaux indicateurs portent sur les dimensions internes de la performance, à l'instar des observations de Farneti et Guthrie (2008). Cette double orientation interne (l'efficience dans les actions) et externe à l'organisation (les effets sur le territoire) rend le contenu de la performance publique relativement large, d'autant qu'elle porte à la fois sur les aspects stratégiques et la gestion courante.

\subsection{L'intérêt d'une mobilisation de la performance globale pour apprécier la performance publique locale}

Le concept de performance globale se développe en Europe avec celui du développement durable, mais ses prémices se trouvent dans des concepts plus anciens tels que la responsabilité sociale et sociétale,abordée aux États-Unis par Carroll dès 1979. La performance globale se définie comme l'agrégation des performances économiques, sociales et sociétales (Capron et Quairel, 2005 ; Reynaud, 2003), ou la satisfaction des performances économiques, sociales et environnementales relativement aux différents acteurs (Doyle, 1994). Dans cette conception, la performance est multi-niveaux (stratégique, tactique, opérationnel) et multi-dimensions (économique, financière, sociale, environnementale, etc.), ce qui pose le problème de son déploiement et une représentation transversale par processus avec une prise en compte des intangibles. Par ailleurs, elle est multi-périodes (court terme, moyen terme, long terme) et multi-acteurs (partenaires, agents, citoyens, contribuable, électeurs, élus, etc.) avec différents enjeux, ce qui entraîne une évaluation multicritère. Ce concept de la performance globale semble correspondre aux situations auxquelles les collectivités sont confrontées dans leurs démarches de performance, même s'il n'est pas toujours mobilisé.

Pourtant, les premières réflexions sur le concept ont débouché sur des outils opérationnels, tels que des adaptations du BSC en Sustainability balanced scorecard (tableau de bord durable) et des adaptations du Navigateur de Skandia (Edvinsson et Malone, 1997) ${ }^{11}$. L'approche du tableau de bord durable nécessite l'intégration du développement durable dans les systèmes de pilotage et de reporting, mais celle-ci peut être totale, partielle ou additive. En ce sens, des indicateurs sur l'environnement peuvent être mis dans chaque axe du BSC, dans un axe dédié à l'environnement, ou bien un ou deux indicateurs sur les axes pertinents.

\footnotetext{
${ }^{10}$ A la différence d'une entreprise visant à favoriser ses apporteurs de capitaux, une collectivité est confrontée à des habitants, contribuables, électeurs, usagers, bénéficiaires, des partenaires institutionnels, économiques et sociaux, dont il est difficile de constituer une hiérarchie des priorités.

${ }^{11}$ Cependant la conception de ce dernier est essentiellement interne (la valeur totale de l'organisation se compose du capital physique, du capital monétaire, du capital structurel et du capital humain) et parait moins pertinente pour les collectivités que les SBSC.
} 
Ainsi, Bieker (2002) suggère d'ajouter une cinquième dimension au Balanced Scorecard, à savoir la dimension sociétale. L'objectif final étant d'élargir le champ des facteurs pris en compte, et de signaler l'importance de ce domaine de performance. La performance finale mesurée reste économique, mais la chaîne de causalité est élargie et les acteurs sont conduits à intégrer les variables sociétales dans le déploiement de la stratégie et à définir les objectifs. Reynaud (2003) propose une représentation de la performance globale sous la forme d'un schéma, qui à défaut d'être un modèle fini pourrait être utile à la réflexion des responsables des collectivités. L'auteur distingue la performance environnementale (réduire la pollution, le gaspillage, les risques d'accidents), la performance économique (efficacité et efficience, notation éthique) et sociétale (diminuer la probabilité d'événements spectaculaires tels que les grèves, améliorer l'équité et justice sociale) concourant à la performance globale.

De même, le modèle de Epstein (1984) avec les mesures organisationnelles interne et externe en quatre axes (l'axe contexte environnemental, l'axe client-citoyen, l'axe des processus de délivrance de service, et l'axe des impacts des services) pourrait intéresser des responsables territoriaux. En effet, on y retrouve les problématiques auxquelles sont confrontées les collectivités et une relation avec les objectifs affichés lors de l'implémentation des démarches de performance (cf. supra, 2.2).

Si ces réflexions sur la performance globale vont dans le sens des travaux menés au sein des collectivités pour mesurer et évaluer les impacts des actions sur leur territoire, et correspondent aux intentions (les objectifs variés affichés des démarches) des dirigeants locaux, il apparaît toutefois que le concept de performance globale n'est pas mobilisé dans son ensemble, et ceci, semble-t-il, pour plusieurs raisons. Tout d'abord, il est vrai que les premières tentatives managériales n'ont pas révolutionné la mesure de la performance car leur mise en place souffre de plusieurs difficultés. Ainsi, Capron et Quairel (2005, p.13) rappellent que «le modèle théorique du Sustainability Balanced Scorecard ne constitue pas un dispositif permettant d'évaluer une performance globale mais il élargit le pilotage économique aux dimensions sociétales ». Cette prise en compte qui permet de rechercher un équilibre entre les finalités des différents acteurs est importante dans les collectivités où l'absence de hiérarchie entre parties prenantes complique la prise de décision. Parallèlement, les limites techniques mises en évidence par les études empiriques et relatives aux difficultés de mise en œuvre du BSC classique sont renforcées par l'introduction de dimensions supplémentaires, les pratiques restent très focalisées sur les indicateurs «faciles » à renseigner, plutôt monétaires (Bieker, 2002), et les aspects plus qualitatifs sont mal pris en compte.

Ainsi, dans les collectivités étudiées, la conception large de la performance affichée dans les objectifs des démarches n'est pas réellement observée à travers les indicateurs faisant l'objet d'un reporting ascendant pour l'équipe de direction, les élus et parfois les habitants. La dimension environnementale est une approche additive, avec un ou deux indicateurs associés à l'axe usager et processus internes, et non par une prise en compte totale avec des indicateurs sur l'environnement dans chaque dimension. Parallèlement, un effort est fait sur la dimension sociétale de la performance publique avec les effets mesurés ou évalués des politiques publiques sur le territoire de la collectivité. Cependant, aucune personne interviewée n'a fait référence au concept de performance globale, et très peu de documents internes mentionnent une référence sur ce point ${ }^{12}$.

\footnotetext{
${ }^{12}$ Le concept est énoncé sans définition dans les rapports annuels du CG 1 et CG 2.
} 
Si l'agrégation des dimensions économiques, financières, sociales, juridiques, sociétales et environnementales pour s'adapter aux finalités du secteur public rend la mesure de la performance (ou des performances) complexe (s), les collectivités semblent avoir intérêt à apprendre des expériences menées quant à la prise en compte de la performance globale. En effet, la performance publique locale intègre différentes dimensions, différentes périodes, différentes cibles et différents enjeux. Or, en retenant une performance multidimensionnelle, ajoutée à l'absence de hiérarchie des parties prenantes dans le secteur public, à la différence d'une entreprise privée travaillant d'abord pour ses apporteurs de capitaux, la collectivité est confrontée au problème du choix stratégique et des objectifs à privilégier. Le caractère multiple du concept incite alors à qualifier les axes d'analyse et à réfléchir à un modèle adapté de représentation de la performance publique locale.

Nous sommes conscients qu'une évaluation globale nécessite dans les collectivités de s'attacher plusieurs problématiques, parmi lesquelles le défi des actifs intangibles, l'intégration du développement durable dans les systèmes de pilotage et de reporting, ou le pilotage des performances au sein d'une chaîne de valeur globale. Cependant des études quantitatives permettant de valider le recours à plusieurs critères dans chaque axe d'analyse de la performance publique peuvent être initiées. Elles peuvent s'appuyer sur les éléments répertoriés dans les CG présentés : les cinq regards pour les cibles, les concepts mesurés pour les enjeux, les objectifs affichés des définitions de la performance pour les dimensions retenues. Or, cette approche quantitative permettrait de limiter les biais de cette étude. En effet, nous avons sélectionné certains conseils généraux par pragmatisme, et les CG, qui appartiennent à une seule strate de collectivité, n'ont fait l'objet que d'une étude sur une année. L'absence de vision longitudinale limite l'analyse du contenu des démarches à un moment donné, d'autant plus que seules des informations déclaratives ont été mobilisées, malgré une triangulation par le nombre d'acteurs rencontrés et de documents consultés.

\section{Conclusion}

L'adoption de la LOLF a permis de multiplier les débats portant sur la modernisation de la gestion publique. Or, si la voie privilégiée à l'échelle nationale est claire, à travers les principes et outils mobilisés, les chemins que les collectivités peuvent emprunter sont, eux, multiples. Nous avons alors cherché à caractériser le contenu des démarches locales de performance publique, en développement depuis le début des années 2000. Nous avons pu observer que des collectivités s'inspirent du cadre national, proposé et retranscrit au travers du guide méthodologique de la LOLF, pour mener leurs démarches et que de nombreuses similitudes dans les outils et les concepts apparaissent, tels que les segmentations en MPA, les rapports de performance, les principes de déconcentration et de responsabilisation, etc.

Toutefois, des divergences peuvent aussi exister en particulier concernant l'appréciation de la performance de manière globale et les modalités de mise en œuvre. Sur le premier point, l'analyse en termes de mimétisme et / ou d'innovation met en évidence l'intérêt du concept de performance globale, dépassant celui privilégié par la LOLF. Les adaptations du BSC en tableaux de bord durables, les propositions sur l'intégration de l'environnement et de la société dans les indicateurs suivis constituent autant d'opportunités de réflexions pour les responsables territoriaux. Sur le second point, celui concernant les modalités, il ressort 
de cette étude que des spécificités dans les démarches de performance des collectivités tiennent à l'implication politique des élus et des fonctionnaires, la création d'interactions entre les différents acteurs, et l'adaptation de la démarche aux caractéristiques des structures. De plus, si le modèle de la BSC semble être utilisé dans les conseils généraux étudiés, les adaptions empruntent parfois aux modèles de performance globale, parfois à la LOLF, pour compléter la représentation et la mesure. Ces adaptations au contexte apparaissent nécessaires dans toutes organisations, mais peuvent prendre diverses formes (instruments plus ou moins décentralisés, participatifs...), selon les facteurs de réussite identifiés.

Cependant, au delà de ces résultats, cette recherche souffre de limites nécessitant des prolongements. Tout d'abord, nous avons mobilisé un nombre limité de collectivités et utilisé des données qualitatives comportant une part d'affichage politique. Par ailleurs, cette étude exploratoire n'analyse pas de manière précise les modalités de mise en œuvre des DPL alors que celles-ci apparaissent spécifiques.

À ce titre, différentes recherches sont envisagées. Tout d'abord, des études quantitatives précisant les dimensions de la performance publique locale apparaissent comme une suite logique à cette recherche exploratoire ; de même que des études confirmatoires sur les divergences entre LOLF et DPL intégrant un plus grand nombre de collectivités. Par ailleurs, nous pensons aussi que des études quantitatives sur les facteurs explicatifs à l'engagement et au développement des DPL soient nécessaires. En outre, la mise en évidence de l'intérêt des recherches portant sur le concept de performance globale est une piste à détailler empiriquement. L'ensemble de ces prolongements nous permettra de mieux circonscrire la notion de performance dans le contexte, qui, si elle connaît des développements instrumentaux et opératoires importants, n'en reste pas moins peu appréhendée de manière conceptuelle et théorique.

\section{Bibliographie}

AMIEL et al., (1998), Management de l'administration, De Boeck Université, Bruxelles.

BARTOLI A., (1997), Le management dans les organisations publiques, Dunod, Paris.

BENZERAFA M., (2007), L'universalité d'un outil de gestion en question : Cas de la Balanced Scorecard dans les administrations de l'Etat, thèse en sciences de gestion, Université de Paris X.

BIED-CHARRETON H., (2006), Performance : nouveau mot d'ordre de la gestion publique, Problèmes économiques 2907, septembre.

BIEKER T. (2002), Managing corporate sustainability with the Balanced Scorecard: Developing a Balanced Scorecard for Integrity Management, Oikos PHD Summer Academy.

BOUCKAERT G. et POLLITT C., (2004), Evaluating public management reforms: a comparative analysis, Oxford University Press, Oxford.

BOYNE G.A., (2002), Public and private management : what's the difference ?, Journal of management studies 39 (1), 97-122.

CAPRON M. et QUAIREL-LANOIZELEE F., (2005), Evaluer les stratégies de développement durable des entreprises : l'utopie mobilisatrice de la performance globale, Journée Développement Durable- AIMS, Aixen-Provence,1-22. 
CARASSUS D., FAVOREU C., (2005), De la performance nationale à la performance locale : étude de 1'application de la LOLF aux collectivités locales, $2^{\mathrm{e}}$ Workshop Ville-Management, décembre.

CARASSUS D.,FAVOREU C.,GARDEY D.,(2010), Les facteurs clés de succès de l'introduction et du développement de la gestion par la performance en collectivité locale, 6e Workshop Ville-Management, juin, Bordeaux.

CARroll A. B., (1979), A Three-Dimensional Conceptual Model of Corporate Performance, Academy of Management Review 4 (4), 497-505.

CHAUVEY J-N., (2005), L'intérêt du Balanced Scorecard dans l'évolution des modes de contrôle des Départements français, Actes du 26ème congrès de l'AFC, Lille.

COLL., (2004), La démarche de performance : stratégie, objectifs, indicateurs. Guide méthodologique pour l'application de la loi organique relative aux lois de finances du 1er août 2001, rapport interministériel, juin. Et version 2007.

CONSEIL DE L'EUROPE, (2005), Rapport sur la gestion des performances au niveau local.

DAMBRIN C., LAMBERT C., SPONEM S., (2005), Contrôle et changement: une perspective néoinstitutionnelle, Actes du 26ème congrès de l'AFC, Lille.

DOYLE P., (1994), Setting business objectives and measuring performance, Journal of General Management $20(2), 1-19$.

DIMAGGIO P. et POWELL W.W., (1983), The iron cage revisited: institutional isomorphism and collective rationality in organizational fields, American sociological review 48, 147-160.

EDVINSSON L. et MALONE M.S., (1997), Le capital immatériel de l'entreprise, Maxima Laurent du Mesnil Éditeur, Paris.

EDWARDS D. et THOMAS J.C., (2005), Developing a Municipal Performance Measurement System: Reflections on the Atlanta Dashboard, Public Administration Review 65 (3), 369-376.

ELKINGTON J., (1997), Canibals with Forks: the Triple Bottom Line of 21st Century Business, Capstone, Oxford.

ENGLISH L. et GUTHRIE J., (1997), Performance information and programme evaluation in the Australian public sector, International Journal of Public Sector Management 10 (3), 154-164.

EPSTEIN P. D., (1984), Using Performance Measurement in Local Government, Van Nostrand Reinhold Company, New York.

FARNETI F., (2006), Balanced Scorecard Implementation in an Italian Local Government, Actes de la Conférence de l'EIASM, Sienne, septembre.

FARNETI F. et GUTHRIE J., (2008), Italian and Australian local governments: balanced scorecard practices. A research note, Journal of Human Resource Costing \& Accounting 12 (1), 4-13.

GIBERT P., (1988), Management public, management de la puissance publique, Politiques et Management Public 4 (2), 89-123.

GIBERT P., (2002). L'analyse de politique à la rescousse du management public ou la nécessaire hybridation de deux approches que tout, sauf l'essentiel sépare, Politiques et Management Public 11 (1), 111-127.

GRAIL C., LESCAILLEZ V., MENUT P. (élèves administrateurs), (2006), Guide sur la performance dans les collectivités territoriales, rapport pour l'AFIGESE-CT, l'ACUF et l'INET, octobre.

GUTHRIE J. et FARNETI F., (2008), GRI sustainability reporting by Australian public sector organizations, Public Money and management 28 (6), 361-366.

HATRY H. P., (1999), Performance Measurement: Getting Results, The Urban Institute Press, Washington.

HLADY-RISPAL M., (2002), La méthode des cas : application à la recherche en gestion, DeBoeck, Bruxelles.

HOOD C., (1995), The new public management in the 1980s : variations on a theme, Accounting, Organizations and Society 20 (2/3), 93-109.

HUTEAU S., (2008), La nouvelle gestion publique locale : LOLF et collectivités territoriales, Le Moniteur, Paris.

JICK T., (1993), Managing Change, Irwin, Homewood, IL.

KAPLAN R.S. et NORTON D.P., (1996), Le tableau de bord prospectif, Pilotage stratégique : les 4 axes du succès, Éditions d'Organisation, Paris. 
KAPLAN R.S., (2010), Conceptual Foundations of the Balanced Scorecard, Working paper, Harvard Business School, Harvard.

KLOOT L. et MARTIN J., (2000) Strategic performance management: A balanced approach to performance management issues in local government, Management Accounting Research 11, 231-251.

LAMBERT A. et MIGAUD D., (2006), La mise en æeuvre de la LOLF, Rapport au gouvernement.

MALTAIS D. et MAZOUZ B., (2004), À nouvelle gouvernance, nouvelles compétences, Revue Internationale de Gestion 29 (3), 82-92.

MARSHALL C. et ROSSMAN G. B., (1999). Designing qualitative research, Sage, Thousand Oaks, CA.

MILES M.B. et HUBERMAN A.M., (2003), Analyse des données qualitatives, De Boeck, Bruxelles.

MÖNCKS J., (1998), La nouvelle gestion publique: boîte à outils ou changement paradigmatique ? in Hufty M. (eds), La pensée comptable, PUF, Paris.

OSBORNE D. et GAEBLER T., (1993), Reinventing Government: How the Entrepreneurial Spirit Is Transforming the Public Sector, Plume Book, New York.

OSBORNE D. et PLASTRIK P., (2000), The Reinventor's Fieldbook, Jossey-Bass, San Fransisco.

PAQUIN M. et TREMBLAY Y., (1997) La réingénierie des processus comme instrument d'amélioration de la performance des organisations publiques in GUAY Marie-Michèle (éd.). Performance et secteur public : réalités, enjeux et paradoxes, Presses de l’Université du Québec, Québec, 115-126.

REYNAUD E., (2003), Développement durable et entreprise : vers une relation symbiotique, 1re Journée AIMS sur le Développement durable, Mai, Angers.

SCOTT W.R., (1995), Institutions And Organisations, Sage Thousand Oaks, CA.

THIETART R.-A. et coll., (1999), Méthodes de recherche en management, Dunod, Paris.

YETANO A., (2009), Managing Performance at Local Government Level: The Cases of the City of Brisbane and the City of Melbourne, Australian Journal of Public Administration 68 (2), 167-181. 